\title{
Las dimensiones de la competencia mediática en estudiantes universitarios españoles
}

\author{
The dimensions of media competence in Spanish \\ university students
}

\section{As dimensões da competência mediática dos estudantes universitários espanhóis}

\author{
Dr. Daniel Aparicio González \\ Profesor asociado \\ (Universidad Complutense de Madrid) \\ https://orcid.org/0000-0002-3242-2203 \\ España \\ Dr. Fernando Tucho \\ Profesor titular \\ (Universidad Rey Juan Carlos) \\ https://orcid.org/0000-0003-4747-0264 \\ España \\ Dr. Rafael Marfil-Carmona \\ Profesor ayudante doctor \\ (Universidad de Granada) \\ http://orcid.org/0000-0001-9688-7088 \\ España
}

Fecha de recepción: 28 de noviembre de 2019

Fecha de revisión: 1 de febrero de 2020

Fecha de aceptación: 8 de abril de 2020

Fecha de publicación: 1 de julio de 2020

Para citar este artículo: Aparicio González , D., Tucho, F. y Marfil-Carmona, R. (2020). Las dimensiones de la competencia mediática en estudiantes universitarios españoles, Icono 14, 18 (2), 217-244. doi: 10.7195/ri14.v18i2.1492 


\section{Resumen}

Los resultados de este trabajo se enmarcan en el proyecto coordinado de $I+D+i$ denominado "Competencias mediáticas de la ciudadanía en medios digitales emergentes: prácticas innovadoras y estrategias educomunicativas en contextos múltiples", desarrollado entre los años 2016 y 2019 en 25 universidades españolas. Concretamente, en el subproyecto "Competencias mediáticas de la ciudadanía en medios digitales emergentes en entornos universitarios". Se han recabado datos entre estudiantes universitarios, a través de cuestionarios y focus groups, para conocer su autopercepción en torno al nivel que poseen en las diversas dimensiones de la competencia mediática, al objeto de extraer claves para la mejora formativa. Según los resultados obtenidos, este colectivo se considera, en su mayoría, competente mediáticamente, algo que no es coherente con la verdadera percepción que demuestra al detallar su autovaloración de cada una de las seis dimensiones principales de la competencia mediática. Tan solo la tecnológica parece suficientemente cubierta, si bien se evidencia una visión muy limitada de la importancia de la producción de mensajes frente a la difusión, así como de la identificación de ideología y los valores o la interacción, entre otros aspectos. Destaca también un bajo grado de asimilación de la dimensión estética y de las implicaciones medioambientales. Esta necesidad de alfabetización mediática y digital coincide con la visión que tiene el profesorado universitario, para el que es evidente esta necesidad formativa. Se reproducen, por tanto, las carencias habituales de la educación mediática tradicional y digital que se han venido detectando en estudios más generales de población.

Palabras clave: Competencia mediática; Educación Mediática; Dimensiones; Estudiantes universitarios; Educación Superior; TIC

\section{Abstract}

The results of this work form part of the coordinated RD\&I project: "Citizens' media competence in relation to emerging digital media: innovative practice and educommunicational strategies in multiple contexts", developed between 2016 and 2019 at 25 Spanish universities. Specifically, the work pertains to the subproject: "Media competence of the citizen with emerging digital media in university settings". Data was collected from university students through questionnaires and focus 
Las dimensiones de la competencia mediática en estudiantes universitarios españoles $\mid 219$

MONOGRÁFICO

groups. We sought to understand their self-perceptions regarding their level of media competence in relation to various dimensions, with the aim of extracting key points in order to improve training provided. According to the results obtained, this group largely considered themselves to be media competent; however, this is not coherent with the true perception revealed following inspection of students' self-ratings for each of the six main dimensions of media competence. Only technology appears to be sufficiently covered. A very limited vision of the importance of producing messages in comparison to their diffusion was evidenced; in addition, values were identified pertaining to the identification of ideology and interaction, among other aspects. A lack of assimilation of the aesthetic dimension and environmental implications is also highlighted. The need for media and digital literacy coincides with the vision held by university teachers, and for this reason the need for training is evident. As a result, it is observed that the common failings detected by more general population studies in relation to traditional media and digital education are being repeated in this sphere.

Key Words: Media competence; Media Literacy; Dimensions; University students; Higher Education, ICT

\section{Resumo}

Os resultados deste trabalho fazem parte do projeto coordenado de $I+D+i$ intitulado "Competências mediáticas dos cidadãos em mídias digitais emergentes: práticas inovadoras e estratégias educomunicativas em múltiplos contextos", desenvolvido entre 2016 e 2019 em 25 universidades espanholas. Especificamente, no subprojeto "Competências mediáticas dos cidadãos em mídias digitais emergentes em ambientes universitários". Os dados foram coletados de estudantes universitários, por meio de questionários e grupos focais, para investigar sua autopercepção sobre o nível que possuem nas várias dimensões da competência midiática e seu desenvolvimento em cada uma delas. Conhecer estes dados relativos à competência mediática e à percepção que os alunos têm dela permite-nos extrair chaves para melhorar a formação no futuro. Os resultados pintam um panorama ainda incerto. Das seis principais dimensões da competência mediática, apenas a competência tecnológica parece estar suficientemente coberta, embora os próprios estudantes exijam formação neste 
MONOGRÁFICO

domínio. No resto, as deficiências habituais da educação tradicional para a mídia em ambientes analógicos parecem ser reproduzidas.

Palavras chave: Competição mediática; Educação mediática; Dimensões; Estudantes universitários; Ensino superior; TIC

\section{Introducción y estado de la cuestión}

La competencia mediática es uno de los ámbitos interdisciplinares de investigación más importantes de cuantos se han desarrollado desde la perspectiva de Educomunicación durante las últimas décadas. A finales del siglo $\mathrm{XX}$, el interés giraba en torno a la capacidad crítica de la ciudadanía frente a fenómenos de influencia masiva como la imagen, la televisión o la publicidad (Aparici y García Matilla, 1987; Ferrés, 1994a y 1994b; Pérez Tornero, 1994). Sin embargo, el acceso a la faceta de producción y difusión marcó una vocación del trabajo heterogéneo e interdisciplinar en la Educación Mediática desde su inicio (Freire, 1970; Kaplún, 1998; Orozco, 1994). Actualmente, esa faceta creativa se diversifica en atención a perfiles concretos, como es el caso del colectivo universitario en este trabajo, en el que se valora el uso de los dispositivos móviles, pero también la propia percepción que tienen los estudiantes de estos medios como herramienta para la enseñanza y el aprendizaje. Si bien la Educación Mediática ha centrado sus esfuerzos investigadores en público escolar o en la ciudadanía en general tradicionalmente, el perfil de estudiantes universitarios de grado, esencialmente de Comunicación o Educación, tiene una enorme importancia tanto en lo relativo a la formación profesional de aquellos colectivos, así como a la detección posible de mejoras formativas en la enseñanza superior. Además, como contenido transversal, la competencia mediática es de gran interés en el proceso formativo de la totalidad de estudiantes universitarios.

Con la consolidación de la cultura digital y las redes sociales, no solo fue necesario adaptar esa mirada crítica a un nuevo contexto de convergencia mediática (Jenkins, 2008), sino que se mantuvo la necesidad del análisis y la comprensión de los contenidos que nos llegaban, pero también que generábamos, a través de las pantallas (Aparici y García Matilla, 2008), con una atención especial a la representación de la realidad por parte de los medios (Aparici, 2010) y a nuevas 
narrativas, como el fenómeno transmedia (Jenkins, Ford y Green, 2015; Scolari, 2013) o el crecimiento de los proceso de gamificación revisados desde la óptica educomunicativa como ludoliteracy (Aranda y Martínez, 2013). Por su perfil como jóvenes en su mayoría, pero también de forma específica por su implicación en la sociedad del conocimiento, el colectivo de estudiantes universitarios adquiere un interés especial.

En Internet, la ciudadanía en general y la juventud en particular han adquirido un perfil activo, gracias a una constante participación en un entorno que sugiere dinámicas de horizontalidad y colectivización de la autoría de los contenidos, haciendo que se revisen y apliquen a la actualidad algunas teorías de finales de los años $70 \mathrm{y}$ de los años 80 del siglo XX, como el concepto de EMIREC (Cloutier, 1975) o prosumer (Toffler, 1980), dos términos que resaltan la inmersión en el proceso comunicacional, pero también en el educativo, si bien la idea de prosumer puede tener más connotaciones mercantilistas (Aparici y García Marín, 2018, p. 77). Se hizo necesaria, además, una revisión crítica del concepto de "multitarea" (0phir, Nass y Wagner, 2009; Spitzer, 2013; Uncapher et al., 2017; Uncapher y Wagner, 2018).

Destaca también el potencial de las tecnologías para impulsar y consolidar lo que se ha denominado "factor relacional" (Marta-Lazo y Gabelas Barroso, 2016), haciendo posible "una nueva evolución individual y colectiva" en lo que son nuevos contextos para la comunicación, de igual forma que para la educación y el aprendizaje (Marta-Lazo, Gabelas Barroso y Marfil-Carmona, 2018, p. 560). Tal y como afirma María Teresa Quiroz, no se trata solo de prepararse o capacitarse, sino de promover un proceso reflexivo para el desarrollo de esas capacidades (2008, p. 47).

En este escenario de transformación, el uso de la tecnología digital es un factor clave. Por ello, la denominada competencia en comunicación audiovisual (Ferrés, 2007) se adaptó al factor digital, una vez que la competencia mediática se consideraba como una "acción educativa inaplazable" (Aguaded, 2012) y, en sí misma, englobaba y ampliaba la consideración de lo audiovisual, dando un paso más y ajustándose al nuevo escenario comunicacional y educomunicativo. Así, esta competencia fue definida en 6 dimensiones diferentes: lenguajes; tecnología; procesos de interacción; procesos de producción y difusión; ideología, valores y estética 
(Ferrés y Piscitelli, 2012). Cada una de esas dimensiones son el resultado de una exploración previa en el ámbito más estricto de la adaptación educomunicativa a los medios digitales (Buckingham, 2009; Pérez-Tornero, 2004) y del análisis y contraste con el currículum formal, una línea de trabajo desarrollada desde la Tecnología Educativa (Area, 2008; Pérez-Rodríguez, Aguaded y Monescillo, 2010; Tondeur, Van Braak y Valcke, 2007) durante unos años en el que el lenguaje pedagógico incorporaba con más intensidad el concepto de competencias (López Herrerías, 2014; Perrenoud, 2004) 1 .

Teniendo en cuenta el análisis realizado por Pérez-Rodríguez y Delgado-Ponce (2012), en el que se valoran también los indicadores para el estudio de la competencia mediática, la consolidación de este proceso de adaptación a la nueva realidad digital ha hecho posible que esta investigación se fundamente en la consideración de las 6 dimensiones básicas, en la que Joan Ferrés y Alejandro Piscitelli dejan clara la flexibilidad de la propuesta y la necesidad de adaptar ese instrumento conceptual a cada situación educativa concreta (2012, p. 77), que divide cada dimensión en la capacidad para el análisis, por un lado, y para la expresión, por otro.

El análisis de la competencia digital es un enfoque que encuentra antecedentes directos en los resultados de investigación aplicados a la ciudadanía en general, como el caso de Andalucía (España) (Aguaded et al., 2011); a diferentes segmentos poblacionales, como estudiantes de Educación Primaria (Ramírez García, Sánchez-Carrero, Contreras-Pulido, 2016) o jóvenes en general (Pereira, Fillol y Moura, 2019), con una destacada presencia de informes de investigación centrados en estudiantes universitarios (Amador y Amador, 2014; Gisbert y Esteve, 2016). Estos trabajos señalan las carencias formativas y a la necesidad de seguir incidiendo en programas y acciones destinados a impulsar la alfabetización mediática en cada uno de los perfiles. En este sentido, el presente estudio se suma a esa línea de trabajo desde su aplicación a un perfil específico cuya etapa formativa tiene una enorme trascendencia social a medio y largo plazo, como es el conformado por los estudiantes universitarios.

En este sentido, hay que especificar que no existen antecedentes de investigaciones similares en las características concretas de esta investigación, como es la 
autovaloración de los estudiantes, pero sí se han analizado aspectos relacionados con la competencia mediática en el contexto educativo universitario, destacando la validación de instrumentos de evaluación (García-Ruiz, Duarte y Guerra, 2014; Pérez-Escoda, García-Ruiz y Aguaded-Gómez, 2018), una línea de trabajo que se une al empleo de las tecnologías para nuevos procesos de enseñanza y aprendizaje como los M0OC (Osuna-Acedo, Marta-Lazo y Frau-Megis, 2018), como ejemplo de las posibilidades colaborativas en el e-learning, además del estudio de la seguridad digital en estudiantes de grados en Educación (Gallego-Arrufat, Torres-Hernández y Pessoa, 2019), entre otros enfoques investigadores.

La preocupación específica por el uso de los dispositivos móviles en estudiantes universitarios (Figueras-Maz, Masanet y Ferrés, 2017; Mateus, Aran-Ramspott, y Masanet, 2017), así como el uso por parte del profesorado con fines docentes (Tyrer, 2019), se enmarca en una línea de trabajo desarrollada durante los últimos años en el ámbito de la didáctica y la tecnología educativa centrada en la Educación Superior, considerando la indudable influencia de la tecnología de nuestro tiempo en los contextos de enseñanza y aprendizaje, que en este trabajo se aborda, de manera específica, desde las dimensiones de la competencia mediática. Es importante especificar que, al tratarse de un contenido tradicionalmente transversal, este trabajo es una primera aproximación a esa autopercepción por parte de los estudiantes, pero que puede servir de punto de partida para el estudio de mejoras formativas en este sentido en el diseño curricular de los grados universitarios.

\section{Metodología}

El objetivo principal de este trabajo es explorar la presencia de la competencia mediática en sus diversas dimensiones entre los estudiantes universitarios, centrándose en su propia autopercepción, es decir, indagando en torno a cómo se percibe este colectivo en cada dimensión y cuál es la relevancia que otorgan a cada una de ellas.

Para abordarlo, la aproximación directa a los estudiantes se ha realizado a través de una doble vía: de un lado, el lanzamiento de un cuestionario estructurado, que fue distribuido a través de una red de profesores y profesoras universitarias por di- 
versas universidades españolas hasta conseguir una muestra autoseleccionada de 897 respuestas. De otro lado, la realización de cuatro focus groups para indagar en mayor profundidad en las percepciones de los estudiantes. Se optó por la técnica del grupo focalizado en lugar del grupo de discusión al tener un cuestionario muy preciso sobre el que obtener respuestas, con una mayor intervención de los moderadores (Ramírez, 2015). La variedad y el factor aleatorio aseguran el trabajo con una muestra significativa, integrando una herramienta cuantitativa y otra cualitativa.

La muestra para el cuestionario fue autoseleccionada, distribuyéndose durante el curso 2017-2018 entre 25 universidades españolas. De las 897 respuestas obtenidas, el $69 \%$ corresponde a mujeres y el 31\%, a hombres. Respecto a titulaciones, el 53\% estaba realizando estudios vinculados a la Comunicación (Publicidad y Relaciones Públicas, Comunicación Audiovisual y Periodismo); el 29\%, estudios de Educación (infantil, primaria, educación social). El 18\% restante pertenece a otras titulaciones, entre las que destacan Marketing, Administración y Dirección de Empresas y Bellas Artes. Se obtuvo representación de estudiantes de todos los cursos: $35 \%$ son de primer curso; $22 \%$, de segundo; $22 \%$, de tercero; y un $16 \%$, de cuarto. Completa la muestra un 5\% de alumnos y alumnas de Máster.

Respecto a los focus groups, el denominado Grupo 1 (G1 en adelante) se realizó en la Universidad Rey Juan Carlos, con un total de siete estudiantes, todos varones, cuatro del Doble Grado en Ingeniería de computadores y Diseño y desarrollo de videojuegos, dos del Doble Grado en Ingeniería Informática e Ingeniería de computadores y uno del Grado en Ingeniería de computadores. El Grupo 2 (G2) se realizó en la Universidad Pablo de Olavide de Sevilla, con seis estudiantes, cuatro varones y dos mujeres, cinco de primer y tercer curso del Grado en Educación Social más un estudiante del Máster de Educación para el Desarrollo, Sensibilización social y Cultura de Paz de esta misma universidad.

El Grupo 3 (G3), por su parte, tuvo lugar en la Universidad de Huelva, con cinco estudiantes del Grado en Educación Primaria, tres mujeres y dos hombres, todos de tercer curso, de entre 22 y 25 años. El Grupo 4 (G4) se desarrolló en Barcelona, con un total de 8 estudiantes, 7 de diversos grados de comunicación y uno de Ciencias Políticas: tres de la Universidad Ramón Llul-Blanquerna, dos de ellos de 
Comunicación y uno de Publicidad y Relaciones Públicas y Marketing; cuatro de la Universidad Pompeu Fabra, tres de ellos de Publicidad y Relaciones Públicas y uno de Ciencias Políticas; y una estudiante de Marketing y Comunicaciones digitales de la Escuela de negocios y dirección EUNCET Business School, adscrita a la Universidad Pompeu Fabra. La referencia a los estudiantes es anónima y se especifica con la inicial E, seguida del número como participante en cada grupo.

Estos resultados complementan la visión de los coordinadores de innovación docente de diversas universidades españolas, que anteriormente habían sido ya entrevistados, en el que se obtuvieron un total de 155 respuestas (Figueras-Maz, Ferrés y Mateus, 2018). El hecho de que los estudiantes entrevistados no pertenezcan a las mismas universidades y grados o grupos asegura una muestra heterogénea de diferentes universidades y titulaciones.

\section{Resultados}

En los resultados de esta investigación ofrecemos, en primer lugar, los datos generales del cuestionario, mostrando una visión más global, para desarrollar posteriormente cada una de las dimensiones de la competencia mediática, sintetizando las principales líneas de autovaloración expresadas por los estudiantes en los focus groups.

\subsection{Respuestas al cuestionario de autopercepción}

Las respuestas al cuestionario permiten afirmar que la autopercepción que los estudiantes tienen de su competencia mediática es moderadamente alta: un $60 \%$ de los estudiantes (540/897) se considera competente en las seis dimensiones, por lo que se perciben a sí mismos como personas competentes mediáticamente. Si damos la opción de restar una de las dimensiones, el porcentaje de los estudiantes que se considera competente en, al menos, cinco de las seis dimensiones, sube hasta el 79\% (710/897). Por lo tanto, podríamos considerar que los estudiantes universitarios que se ven a sí mismos como bastante competentes mediáticamente son casi 8 de cada 10, un porcentaje bastante elevado. Sólo 5 de las 897 personas se consideran no competentes en todas las dimensiones, lo que representa un valor residual. 
MONOGRÁFICO

Atendiendo al sexo, podemos observar que existe bastante equilibrio entre hombres y mujeres: el $62 \%$ de los varones encuestados se considera competente en todas las dimensiones junto a un 59\% en el caso de las mujeres. Sí encontramos más diferencias si atendemos a los estudios de los alumnos dentro de la muestra obtenida: los que más competentes se consideran, en consonancia con su especialidad, son los estudiantes de Bellas Artes (un $81 \%$ de los que han respondido al cuestionario, aun siendo una muestra pequeña de 21 en total). Los que menos, los estudiantes de Psicología, un 45\% (también con una muestra baja de un total de 33 respuestas). Se observan también diferencias propias del perfil entre el alumnado de grados de Comunicación, donde un $65 \%$ se considera competente en todas las dimensiones (256/394), frente a un 54\% en estudios de Educación (132/243).

Estos futuros docentes, como se reflejó en alguno de los focus groups, son conscientes de que están preparándose para una profesión sometida a una presión para una mayor "tecnologización": "los niños vienen alfabetizados, el modelo de escritura debe cambiar, la forma ha cambiado, la escuela no quiere ver. Los niños no es que no lean, sino que ha cambiado su forma de leer y de escribir" (G3-E2). Además, estos estudiantes consideran que se enfrentan a generaciones que se suponen aún más nativas digitales que la suya: "los niños son nativos tecnológicos, sabrán más que nosotros, no podemos hacer un curso al año obligatorio" (G3-E4). A pesar de ello, y a pesar del incremento de asignaturas específicas sobre tecnologías en los grados de educación, estos alumnos no parecen estar satisfechos con la formación que reciben al respecto: "la competencia digital es una exigencia laboral pero en la institución no nos la enseñan" (G3-E2); "no se educa ni hay asignatura, es primordial, pero es secundario en la universidad" (G3E4); "no te enseñan como profesor a enseñar la pizarra digital" (G3-E1).

La alta autopercepción es coherente con la respuesta dada cuando se les pregunta por cuál consideran que es su nivel general de habilidad/conocimiento en el uso de las TIC: un 90\% (803/897) se situaba en un nivel avanzado (401) o medio (402). 32 se consideraban como "expertos", apenas un 4\%, frente al 6\% (58) que se considera "principiante". Por su parte, 4 personas marcaron la opción de "nulo", aunque luego sí decían mostrarse competentes en algunas de las dimensiones. La siguiente tabla muestra el porcentaje de estudiantes que se considera competente en cada una de las dimensiones estudiadas, presentadas en orden de mayor a menor: 


\begin{tabular}{|l|l|}
\hline \multicolumn{1}{|c|}{ Dimensión } & Porcentaje \\
\hline Tecnología & $93 \%(831)$ \\
\hline Lenguajes & $89 \%(798)$ \\
\hline Procesos de interacción & $89 \%(794)$ \\
\hline $\begin{array}{l}\text { Procesos de producción y } \\
\text { difusión }\end{array}$ & $88 \%(791)$ \\
\hline Ideología y valores & $85 \%(760)$ \\
\hline Estética & $83 \%(748)$ \\
\hline Todas las dimensiones & $60 \%(540)$ \\
\hline
\end{tabular}

Tabla 1: Porcentaje de estudiantes que se considera competente en cada una de las dimensiones. Fuente: Elaboración propia.

En el estudio de una generación que es considerada como "nativa digital", la dimensión tecnológica es la que concita mayor unanimidad: un 93\% se considera competente mediáticamente. El cuestionario presentaba esta dimensión de la siguiente forma: "Soy capaz de utilizar las tecnologías y moverme en diferentes entornos digitales".

Ya por debajo del 90\%, las dimensiones de "Lenguajes" ("Soy capaz de analizar y producir contenido con diferentes códigos (sonoro, audiovisual, transmedia"), "Procesos de interacción" ("Soy capaz de cuestionarme críticamente el contenido de consumo a través de dispositivos móviles") y "Procesos de producción y difusión" ("Soy capaz de producir y distribuir elementos a través de dispositivos móviles (memes, posts, mensajes, vídeos...)" son las que siguen a la tecnología en la escala de competencia.

Quedan un poco más alejadas las dimensiones de "Ideología y valores" ("Soy capaz de crear contenido con determinados valores sociales"), con un 85\%, y "Estética" ("Soy capaz de valorar y crear contenido con calidad estética y creatividad a través de dispositivos móviles"), con un $83 \%$ de estudiantes que se considera competente en este ámbito. Los focus groups nos permiten profundizar un poco más en estos datos, desarrollando estos contenidos según el orden de las correspondientes dimensiones. 
MONOGRÁFICO

\subsection{Dimensión "Tecnologías"}

Los participantes en los focus groups vinculan esta alta competencia autopercibida en la dimensión tecnológica a un doble factor. De un lado, la habituación al uso de los dispositivos y la fuerte presencia que tiene en su vida diaria: "La tecnología forma parte de nosotros. Es una obligación. No comprendo la vida sin la tecnología" (G4-E2). Hasta el punto de sentir la "multitarea" como parte de su "generación" (G4-E3).

De otro lado, vinculan esta alta preparación autopercibida en lo tecnológico a la necesidad para sus estudios y su desarrollo profesional, cuestión a la que dedican gran espacio en sus discursos, al ser grupos que se desarrollaron en su entorno universitario:

Me gusta mucho la tecnología, tengo Iphone, tengo Mac. Por mi trabajo utilizo muchísimo el ordenador. Estuve trabajando un tiempo con temas de comunicación y demás. Y ahora, estudiando me da mucha más utilidad tener todo conectado (G2-E5).

En esta carrera debes dominar programas como el Indesign. Debes dominarlo a la perfección. Hay asignaturas que son todas en el ordenador. Yo al principio no sabía, pero se aprende. No puede salir al mundo laboral sin dominar las tecnologías a la perfección (G4-E1).

Con todo, también existe algún caso que se resiste a esta lógica tecnológica, representando, podemos intuir, a una corriente minoritaria pero existente como expresa este estudiante de publicidad:

Soy romántico, tradicional. No sé utilizar un Mac [...] No me gusta que te impongan esa lógica tecnológica [...] Me dan ganas de irme de la pantalla. De irme a la montaña. Me siento muy incompetente. No, no soy incompetente. Puedo hacer una campaña, pero llega un momento que me siento inútil. No hace que me vaya mal, pero me avergüenzo. Hay un amor odio con la tecnología (G4-E4). 
Las dimensiones de la competencia mediática en estudiantes universitarios españoles $\mid 229$

MONOGRÁFICO

Una corriente que quizá vaya perdiendo adeptos, como expresa este otro alumno:

Antes también era tradicional, romántico, pero me he adaptado a la tecnología. Domino todo que le tengo que dominar. Me considero competente. En redes sociales y todo. Por lo que estudio me encamina un poco. Soy un nostálgico que quiere hacer guiones, pero que también tendré que dominar hacer una campaña a través de una red social, una página web o un producto transmedia (G4-E5).

\subsection{Dimensión "Lenguajes"}

Los estudiantes, en una gran mayoría (89\%), se mostraban en el cuestionario como competentes en la dimensión de los lenguajes, entendida como la capacidad de analizar y producir contenido con diferentes códigos (sonoro, audiovisual, transmedia). Sin embargo, en los grupos de discusión fue difícil conseguir un mayor detalle explicativo sobre esta cuestión. Quizá por la naturalización que parecen vivir al respecto: "Estamos tan expuestos al lenguaje audiovisual que estamos entrenados, tenemos la mente estructurada" (G1-E5). Este factor conllevaría una naturalización también en su uso: "Hoy cualquiera puede hacer cosas que hace diez años solo veías en televisión, incluso un niño de 7 años" (G1-E1). Aun reconociendo que la habilidad puede estar más inclinada hacia el ámbito del análisis que de la expresión: "Más capaces de analizar que de crear. Personalmente se me da mejor expresarme en lenguaje escrito" (G1-E5).

Resulta llamativo cómo, quizá por "deformación profesional", los estudiantes de grados de Educación asocian la cuestión automáticamente con los efectos que tienen los nuevos códigos del mundo tecnológico sobre nuestro lenguaje escrito, obviando la vertiente audiovisual y multimedia de esta dimensión:

Con respecto al lenguaje intento no acortar las palabras, porque me daba cuenta de que al escribir ya estaba teniendo incluso faltas de ortografía y eso sí lo estoy corrigiendo (G2-E5).

Incluso reflexionan sobre las consecuencias más profundas que tiene este hecho en este contraste entre la comunicación mediada y la comunicación presencial 
Se utilizan mucho los emoticonos -las caritas- para expresar sentimientos. Pienso que, aunque lo pongan en los mensajes, como no estamos hablando directamente con ellas sino por el móvil pueden utilizarlo lo sientan o no (G2-E6).

Saben comunicarse por móvil y no en un organismo público o en una explicación oral. Se pierde las habilidades sociales, no se mira a los ojos (G3-E2).

Aunque hay también quien le ve el lado positivo: "a mí me ha beneficiado a la hora de tomar apuntes. Por ejemplo, el "para" es "xa". Tengo mis propios códigos" (G3-E3).

\subsection{Dimensión "Procesos de interacción"}

Se presenta la dimensión de "Procesos de interacción" a los participantes en los grupos como la capacidad de cuestionarse críticamente el contenido que se consume a través de dispositivos tecnológicos, pero también como la capacidad de valorar, seleccionar, revisar y autoevaluar la propia dieta mediática. Y es esta segunda cuestión, vinculada directamente a la "adicción a las tecnologías", la que parece tocarles más directamente, activándose en algunos casos desde la autocrítica, utilizando la ironía y el humor para hacer ver su enganche a los dispositivos: "Prefiero no pensar (risas)" (G1-E2); "Puedo dejarlo cuando quiera (risas)" (G1-E5). Desde el reconocimiento de esa realidad de dependencia, esto es especialmente claro en el grupo formado por estudiantes de ingenierías:

Te tiras días perdidos entre juegos, vídeos, que sabes que son basura. Tienes conciencia de ello, pero sigues (G1-E5).

Lo típico de un vídeo más, un capítulo más, y acabar a las tres de la mañana (G1-E2)

Esta autovaloración no solo se produce en el perfil de estudiantes señalado, sino que también se encuentra en el alumnado de Educación:

Me levanto y ya estoy mirando el móvil (G2-E2). 
Las dimensiones de la competencia mediática en estudiantes universitarios españoles | 231

MONOGRÁFICO

Todo el mundo tiene el despertador en el móvil, desde el minuto 1 ya empezamos a ver si tenemos mensajes, quién ha publicado o subido algo. Todo eso antes de levantarte, tumbado en la cama (G2-E3).

Junto a ese "impulso de confesión", se remarcó también la utilidad de las tecnologías móviles, sin dejar de tener presente la autocrítica, especialmente al sentirse increpados por sus adultos o por los medios de comunicación respecto a su enganche a dispositivos:

Es que no es sólo entretenimiento... La facilidad de contactar con los demás, de buscar cosas... Cuando estoy sin móvil entro en angustia, y pienso: lo que me estoy perdiendo... ¿Cómo le pregunto esto a este? Tengo que buscar algo en Google, y estoy fuera de casa, ¿cómo lo hago? (G1-E4).

Ya no usamos agenda, sino el calendario web... Mapas de carretera, antes mis padres compraban cada año, desde que hay gps, no compramos (G1-E5).

Siempre llevo conmigo mi ordenador porque tengo todo ahí. Además, ahora que estoy aquí para ponerme en contacto con mi mamá, con mis amigos en Italia. El ordenador me organiza la vida. Hay aplicaciones como el Calendar o, por ejemplo, una sobre la regla que es muy importante: este es tu calendario, de aquí a aquí, fertilidad, de aquí a aquí, regla (G2-E6).

Se sugirió la idea de que los adultos han generado ese contexto tecnológico $\mathrm{y}$, ahora, les culpan por estar inmersos en él, predicando ellos mismos incluso un uso racional de los dispositivos:

$\mathrm{Si}$, por ejemplo, los padres usan la tablet para que el niño se calle, ¿cómo no vamos a ser dependientes de mayores? Con nosotros usaban la tele, no sé por qué tanto alarmismo. Solo ha cambiado el medio (G1-E3).

Se destacó algún concepto vinculado a los "procesos de interacción", como la capacidad crítica, de la que hablan aquellos que parecen haber recibido algo de formación sobre ella en sus estudios, con alguna referencia a la privacidad, incluyendo el uso de esas redes sociales en clase: 


\section{MONOGRÁFICO}

Ahora quizás estoy siendo más selectiva con el tema de la información y me cuesta mucho encontrar artículos que me sirvan, leo sobre los autores, y en internet millones de veces dan información que no sabes de quién es... somos más críticos (G2-E5).

Sí, soy bastante más crítica con las apps y los programas sobre todo con Google y Facebook. Si veo algo en internet no confío en ello, no me quedo con las tonterías, leo toda la página y decido en una búsqueda y menudeo con la que me interesa (G2-E6).

Whastsapp te dice que los menores de 13 años no pueden utilizarla, o eso dicen. Marcas una casilla y ya no puedes utilizarla, pero eso quién te lo asegura. No vale para nada (G2-E1).

Un profesor también hizo un comentario sobre la vida privada de una alumna y su relación. No se deben realizar comentarios de la vida privada. No estamos al mismo nivel. Debe haber unos límites (G1-E2).

Entre los alumnos de Educación, la preocupación deriva también hacia el uso que hacen los niños y niñas y la formación que reciben:

El uso del móvil en Primaria hace que el alumno que no es conocido en redes sociales pierda valía, y surge el bullying (G3-E3).

No se deben prohibir las redes sociales sino educar para su uso. No solo tienen responsabilidad los niños sino también los padres. Explicarles ventajas, pero también inconvenientes (G3-E1).

\subsection{Dimensión "Procesos de producción y difusión"}

Aunque esta dimensión hace referencia a la capacidad tanto de producir como de difundir contenidos mediáticos, y los estudiantes afirmaron sentirse competentes para ello en el cuestionario, en los grupos de discusión el debate se inclinó más hacia el ámbito de la difusión: 
Las dimensiones de la competencia mediática en estudiantes universitarios españoles $\mid 233$

MONOGRÁFICO

Los móviles... tener redes sociales es fundamental como por ejemplo buscar piso, con una imagen que subas ya puedes tener compañeras de piso. La difusión es ligera y rápida (G3-E3).

Recientemente he compartido una campaña que se llama "los colores de la piel", que se llevó cabo hace varios años. La he descubierto hace poco y buscando ideas la he compartido y he tenido 1.020 compartidos (G3-E4).

Destacó el comentario en torno a la poca relevancia e incluso peligrosidad de los contenidos que triunfan en las redes y del poder de los influencers:

Sobre los influencers, pueden ser radicales y lo ves peligroso para algunos chavales que no tienen una opinión formada (el Rubius, por ejemplo, que todo lo que dice es la leche, se puede cargar un juego porque él dice que es una mierda) (G1-E2).

Veo peligroso qué rápido se crean modas y se difunden: la ballena azul, por ejemplo, un juego que básicamente acababa en suicidio. Y sí, está el control parental, pero luego lo ven con amigos y demás (E1-G5).

Y, como en el apartado anterior, se termina aludiendo al papel de los padres y su sustitución por otros agentes normativos:

Hay tanta información que no se puede controlar... Antes hacíamos más caso a los padres. Tus padres te decían, si un amigo tuyo se tira por un puente, ¿tú le sigues? Y ahora los niños no hacen tanto caso a sus padres, son los influencers o lo que ven en Internet (G1-E6).

\subsection{Dimensión "Ideología y valores"}

A partir de estas dimensiones, el cuestionario mostraba porcentajes algo menores respecto a la autopercepción de competencia, aunque se situaba en un $85 \%$ en lo relativo a "ideología y valores". Los grupos de discusión muestran la existencia de los lugares comunes habituales en este terreno, aunque llevándolo también hacia las redes sociales: 


\section{MONOGRÁFICO}

Muchos medios de comunicación solo piensan en tener mayor cantidad de lectores y sacar beneficios que no en dar una información adecuada (G2-E2).

No todo el mundo tiene consciencia, a veces la gente lo toma como sentencia, lo que sale en los medios, dicen, es la verdad. La formación es lo que te dirá que no es fiable, un periódico da la noticia según la ideología (G3-E2).

En Facebook salen siempre muchas informaciones, cuando salen noticias nuevas siempre son muy rápidas. Vamos, que la gente se entera más con estos medios, pero no sé si detrás hay algo, por ejemplo, dejan pasar algunos mensajes y otros los ocultan o no lo ponen (G2-E6).

Algunas reflexiones, más profundas, se ligaban directamente a sus experiencias en torno al contenido ideológico:

Las redes sociales, la mayoría de las veces, no las usamos para ver las opiniones de los otros sino para que nuestra opinión sea la más importante. Pongo mi comentario para que mi comentario lo vea todo el mundo y yo tenga más seguidores que nadie. Busco llamar la atención y destacarme sobre los demás (G2-E1).

Creo que las redes sociales han facilitado que se tenga una ideología global y una propia. Tiene que haber un referente, una persona referente a la izquierda, una a la derecha. Bueno, y si yo estoy de acuerdo con aspectos tanto de la derecha como de la izquierda, ¿qué? ¿Soy un raro si no me desmarco para ningún lado? (G2-E1).

\subsection{Dimensión "Estética"}

Llegamos a la dimensión que menor unanimidad concita, aunque el porcentaje de los que se consideran competentes sigue siendo de un $83 \%$ en el cuestionario. Sin embargo, a tenor de los comentarios obtenidos en los focus groups, es legítimo dudar de esta autopercepción. Frente a una dimensión que se les presenta como “la capacidad de analizar, valorar y crear mensajes audiovisuales desde el punto de 
vista de la innovación formal y temática y la educación del sentido estético", los estudiantes aportan interpretaciones muy particulares, vinculadas a contenidos tan dispares como los ganchos para conseguir "likes" o la estética de los propios dispositivos, lo que indica cierta dificultad para la propia comprensión de este concepto de la competencia mediática y digital:

La miniatura del vídeo, que está pensada en que sea bonita para llamar la atención y que entres al vídeo, el típico de la mujer medio desnuda para que entres (G1-E2).

Aquí hablamos de la estética, pero yo nunca he sabido elegir entre los móviles (G2-E6).

\subsection{Cuestión medioambiental}

Además de las seis dimensiones de la Educación Mediática reseñadas, tal y como se viene recogiendo en trabajos previos (Tucho, Masanet y Blanco, 2014), hemos considerado interesante incluir una cuestión añadida por su relevancia social y, en concreto, por su conexión con la Educación Mediática, como es el valor medioambiental, dado el impacto material que tienen las tecnologías sobre nuestro entorno (Tucho, Vicente-Mariño y García de Madariaga, 2017).

Esta cuestión se introdujo en los grupos de discusión. Como ocurre con las implicaciones sociopolíticas, el factor medioambiental no parece estar en los primeros niveles de preocupación de los estudiantes respecto al uso de nuevas tecnologías y aplicaciones móviles. Muestran algún conocimiento sobre esta temática, aunque también cierta indiferencia, con discursos poco elaborados y autoexculpatorios:

Por lo general no se le da importancia, porque a la gente no le afecta directamente... Puedes estar concienciado, pero al final te dejas llevar (G1-E1).

La gente no lo piensa, todo el mundo es consciente pero no lo pensamos. Si sólo lo hago yo, qué más da (G1-E3). 
Los móviles vienen de China y allí sabemos que las medidas medioambientales son nulas (G1-E7).

Los fabricantes de esas tecnologías, ¿por qué no están encima? Igual que los supermercados se preocupan ahora por lo ecológico, ¿por qué no está eso en las empresas de tecnología? (G1-E5).

También se pueden encontrar discursos algo más centrados en el vínculo entre tecnología y medio ambiente:

Creo que no es sostenible. No vemos cómo se hace realmente, de dónde vienen las piezas, cómo se fabrica, que es mejor no pensarlo, pero hay que pensarlo (G2-E5).

A mí me da miedo dónde va todo el material, con lo poco que duran los móviles, las pantallas y demás... Los tenemos en un cajón un tiempo, pero luego hay que tirarlos. Por ejemplo, ordenadores que a los 8 años están completamente obsoletos (G1-E4).

De esta forma, las opiniones expresadas recogen, con mayor claridad o diversidad de criterios, según el caso, el posicionamiento de estudiantes universitarios y su autopercepción ante cada una de las dimensiones de la competencia mediática, con el factor añadido de la responsabilidad y concienciación de las implicaciones que conectan la mediación tecnológica y el medio ambiente.

\section{Discusión y conclusiones}

El análisis de las respuestas de los estudiantes muestra un panorama que apunta, salvo alguna excepción, carencias relevantes en la adquisición de competencias de la educación mediática, pero una autovaloración muy positiva. Si bien los resultados no pueden considerarse extrapolables a toda la realidad universitaria, la tendencia manifestada es válida de cara a formular hipótesis para futuras investigaciones, lo que hace posible realizar un balance positivo de la integración de metodologías cuantitativas y cualitativas en este estudio, señalando la posibilidad de futuras mejoras, tanto en muestra como en diversidad o nivel de profundización. En este sentido, es importante seguir escuchando al alumnado, atender sus 
Las dimensiones de la competencia mediática en estudiantes universitarios españoles $\mid 237$

MONOGRÁFICO

inquietudes, siendo interesante la comparativa con las impresiones del profesorado, destacando el valor de estudiar un colectivo de estas características, por la importancia y trascendencia social en el futuro.

Conviene remarcar, de entrada, la elevada autopercepción que los estudiantes tenían sobre su competencia mediática en el cuestionario realizado. Sin embargo, en los focus groups se hacen evidentes algunas carencias y la consolidación de tópicos y lugares comunes que no son la mejor vía para la alfabetización mediática. De igual forma, esta investigación confirma los bajos resultados en competencia mediática que el grupo de jóvenes viene obteniendo desde las primeras evaluaciones globales de referencia sobre competencia mediática de la población española (ITE, 2011). Los resultados son coherentes, por tanto, con las necesidades de procesos formativos y de una cultura educomunicativa en entornos de aprendizaje formal y no formal, que son las propuestas señaladas en las investigaciones referenciadas en el estado de la cuestión, dando un paso más en la autovaloración de esas competencias (López Herrerías, 2014), así como en el detalle de las dimensiones planteadas y desglosadas (Ferrés y Piscitelli, 2012; Pérez-Rodríguez y Delgado-Ponce, 2012), pero aplicadas a un colectivo específico. Esta investigación se suma, por tanto, a la línea de demanda formativa en lo relacionado con la competencia mediática y digital, reforzando las necesidades formativas en este aspecto por parte del alumnado universitario, que ya habían sido evidenciadas en perfiles más generales de la población joven (Pereira, Fillol y Moura, 2019), y aportando el componente específico de la autopercepción.

Sus conclusiones, además, son coherentes con el avance de entrevistas a profesorado de grados universitarios en el contexto del mismo proyecto $\mathrm{I}+\mathrm{D}+\mathrm{i}$, en las que se destaca esa misma visión de la competencia mediática de sus estudiantes, es decir, coinciden con el hecho de señalar la necesidad formativa en esta cuestión por parte de su alumnado y, por lo tanto, con los resultados de este trabajo. Se establece, por tanto, un reto a corto plazo para futuras investigaciones, como es profundizar en perfiles como Comunicación o Educación, grados destinados a la formación de profesionales con una función mediadora en este aspecto, valorando cuáles son las mejoras específicas que requiere el currículum universitario, adaptando correctamente el componente transversal de este tipo de contenidos, aplicable sin duda a la totalidad de los grados universitarios. 
La competencia más dominada por los estudiantes sería la tecnológica, una cualidad que, con reservas, podría entenderse propia de nativos digitales, que son diestros en el manejo de la tecnología móvil. Sin embargo, no se debe desatender el hecho de que los jóvenes demandan formación en lo tecnológico, por considerarlo un aspecto crucial para su futuro profesional, especialmente los futuros docentes. Por su parte, habría mucho camino por recorrer en la dimensión "Lenguajes". Se autoperciben como competentes, pero no deja de ser paradójico que entre las respuestas asociaran la dimensión "Lenguaje", mayoritariamente, a cuestiones verbales de lenguaje escrito (faltas de ortografia, sintaxis...), ignorando otras posibilidades de los lenguajes digitales: creación de discursos con imágenes, vídeos, gráficos, etc. No obstante, le dan importancia a la dimensión "Lenguajes" por considerarla una necesidad.

En cuanto a la dimensión "Procesos de interacción", aún hay carencias relevantes, pero se intuyen tímidos avances. Los estudiantes admiten reconocer situaciones de riesgo, como la dependencia tecnológica o el estrés ante la sobresaturación de información. Lo ideal sería considerar los factores positivos y negativos, valorando tanto las condiciones ventajosas de estas tecnologías, como la ubicuidad y el feedback que favorecen, como las preocupantes: infoxicación, phubbing... Sin embargo, se destaca la dependencia, ya que muchos afirman "no poder vivir sin el móvil".

Algo similar puede aplicarse a la dimensión "Procesos de producción y difusión". Los estudiantes parecen competentes a la hora de detectar el riesgo de la tecnología móvil, que impone modas y estilos de vida; o el peligro de que ciertos influencers marquen tendencias. Sobre todo, celebran la posibilidad de grabar y producir procesos de aprendizaje. Sin embargo, aunque no lo conceptualicen como tal en sus respuestas, sí cabe entender que son competentes en cuanto a procesos de difusión, al menos en su vertiente más práctica, pues la trabajan asiduamente al difundir y recibir contenidos de clase, crear grupos de difusión entre compañeros, etc. Lo que no se cuestionan tanto es la procedencia de la información que reciben y otras potencialidades que brinda la tecnología móvil, como pueden ser la de conectar universidades o divulgar conocimiento. 
La dimensión "Ideología y valores" parece trabajarse en experiencias aisladas, casi siempre vinculadas a asignaturas de comunicación. Es decir, se hace un uso instrumental de la tecnología para hacer, en el mejor de los casos, lecturas críticas de lo que los medios, tradicionales o no, proyectan, pero se dan dos hándicaps importantes: esto ocurre mayoritariamente en asignaturas vinculadas a comunicación, y, por otro lado, no se explican las derivaciones o influencias ideológicas, la presencia de valores en la tecnología en sí. No obstante, el sentido crítico de la valoración de los influencers parece evidenciar cierta resistencia o toma de conciencia en torno a la manipulación.

La dimensión "Estética" y la cuestión medioambiental son áreas prácticamente anecdóticas en los procesos educativos universitarios según los datos obtenidos en la investigación. Una vez más, ambas se asocian a contenidos curriculares específicos en determinadas asignaturas, pero no se aprecia una preocupación latente por trabajar estas dimensiones. Mientras la estética se analiza desde una perspectiva muy limitada, el medio ambiente no es un aspecto que se vincule especialmente a tecnologías y competencia mediática.

Por último, es importante remarcar que es necesario perseverar en la escucha a los estudiantes para seguir detectando carencias en competencia mediática que, abriendo el espectro muestral a otras titulaciones, cabe pensar que puedan ser mayores en el panorama universitario global. Además, puede afirmarse que es pronto para sacar conclusiones relevantes de una tecnología móvil de reciente inclusión en las aulas, que necesitará la perspectiva del tiempo para conocer su valor y aportación dentro y fuera de los recintos universitarios. Han pasado apenas diez años desde la aparición y posterior masificación del Smartphone, por lo que es normal que aún no exista una interiorización ni competencias plenamente desarrolladas. En ese sentido, es importante seguir investigando, desarrollando proyectos de innovación docente y evaluando las dinámicas innovadoras, pero desde la perspectiva de un alumnado que está formándose para hacer posible la transformación de la sociedad. 


\section{Fuentes de financiación}

Investigación realizada con apoyo del proyecto I+D: "Competencias mediáticas de la ciudadanía en medios digitales emergentes en entornos universitarios" (EDU2015-64015-C3-2-R)

\section{Referencias}

Aguaded, J.I. (2012). La competencia mediática, una acción educativa inaplazable. Comunicar, 20(39), 7-8. https://doi.org/10.3916/C39-2012-01-01

Aguaded, J.I., Ferrés, J., Cruz Díaz, M.R., Pérez Rodríguez, M.A., Sánchez Carrero, J. y Delgado-Ponce, A. (2011). El grado de competencia mediática en la ciudadanía andaluza. Huelva: Grupo Comunicar. Recuperado de http://hdl. handle.net/10272/6892

Amador, P., \& Amador, J. (2014). Academic advising via Facebook: Examining student help seeking. The Internet and Higher Education, 21, 9-16. http:// dx.doi.org/10.1016/j.iheduc.2013.10.003

Aparici, R. (Coord.) (2010). La construcción de la realidad en los medios de comunicación. Madrid: UNED.

Aparici, R. y García-Marín, D. (2018). Prosumidores y emirecs: Análisis de dos teorías enfrentadas. Comunicar, 26(55), 71-79. https://doi.org/10.3916/C552018-07

Aparici, R. y García Matilla, A. (1987). Lectura de imágenes. Madrid: Ediciones de la Torre.

Aparici, R. y García Matilla, A. (2008). Lectura de imágenes en la era digital. Madrid: Ediciones de la Torre.

Aranda, D. y Martínez, S. (2013). Ludoliteracy. Media literacy in gaming. En D. Aranda, A. Creus y J, Sánchez-Navarro (eds.), Educación, medios digitales y cultura de la participación (pp. 47-66). Barcelona: UOC.

Area, M. (2008). La innovación pedagógica con TIC y el desarrollo de las competencias informacionales y digitales. Investigación en la Escuela, 64, 5-17. https://idus. us.es/handle/11441/60859 
Buckingham, D. (2009). The future of media literacy in the digital age: some challenges for policy and practice. En P. Verniers (Org.), EuroMeduc: Media Literacy in Europe: Controversies, Challenges and Perspectives (pp. 13-23). Bruselas: EuroMeduc. Recuperado de https://bit.ly/2YAzIHt

Cloutier, J. (1975). L'ère d'EMEREC ou la comunication audio-scripto-visuelle à l'heure des self-media. Montreal: Les Presses de L’Université de Montreal.

Ferrés, J. (1994a). Televisión y Educación. Barcelona: Paidós.

Ferrés, J. (1994b). La publicidad. Modelo para la enseñanza. Barcelona: Paidós.

Ferrés, J. y Piscitelli, A. (2012). La competencia mediática: propuesta articulada de dimensiones e indicadores. Comunicar, 19(38), 75-82. https://doi.org/10.3916/ C38-2012-02-08

Figueras-Maz, M, Masanet, M.J, y Ferrés, J. (2017). Mobile devices in higher education: A pending issue in multidimensional media literacy. Catalan Journal of Communication y Cultural Studies, 9(1), 135-144. https://doi.org/10.1386/ cjcs.9.1.135 1

Figueras-Maz, M., Ferrés, J. y Mateus, J.C. (2018). Percepción de los/as coordinadores/as de la innovación docente en las universidades españolas sobre el uso de dispositivos móviles en el aula. Revista Prisma Social, (20), 160-179. Recuperado de https://bit.ly/2psvnGw

Freire, P. (1970). Pedagogía del oprimido (J. Mellado, trad.). México: Siglo XXI. Gallego-Arrufat, M.J, Torres-Hernández, N. y Pessoa, T. (2019). Competencia de futuros docentes en el área de seguridad digital. Comunicar, 27(61), 57-67. https://doi.org/10.3916/c61-2019-05

García-Ruiz, R., Duarte, A., y Guerra, S. (2014). Propuesta de un instrumento de evaluación para medir el grado de competencia mediática en la etapa de educación infantil. Pixel-Bit, 44, 81-96. https://doi.org/10.12795/pixelbit.2014.i44.06

Gisbert, M. y Esteve, F. (2016). Digital Leaners: la competencia digital de los estudiantes universitarios. La cuestión universitaria, (7), 48-59. Recuperado de https://bit.ly/2NbRr1V

ITE (Instituto de Tecnologías Educativas) (2011). Competencia Mediática. Investigación sobre el grado de competencia de la ciudadanía en España. Subdirección General de Documentación y Publicaciones del Ministerio de Educación del Gobierno de España. Recuperado de https://bit.ly/2Wz3rmf 
Jenkins, H. (2008). Convergence culture: La cultura de la convergencia de los medios de comunicación (P. Hermida Lazcano, trad.). Barcelona: Paidós.

Jenkins, H., Ford, S. y Green, J. (2015). Cultura Transmedia: La creación de contenido y valor en una cultura en red (X. Gaillard Pla, trad.). Madrid: Gedisa. Kaplún, M. 1998. Una pedagogía de la comunicación. Madrid: Ediciones de la Torre. López Herrerías, J.A. (2014). Enseñar y aprender competencias. Málaga: Aljibe. Marta-Lazo, C. y Gabelas Barroso, J.A. (2016). Comunicación digital. Un modelo basado en el Factor R-elacional. Barcelona: UOC.

Marta-Lazo, C., Gabelas-Barroso, J.A. y Marfil-Carmona, R. (2019). El Factor R-elacional y el ecosistema 3.0: nuevas conectividades, nuevas saturaciones. En L.M. Romero-Rodríguez y D.E. Rivera-Rogel (coords.), La comunicación en el escenario digital: actualidad, retos y prospectivas (pp. 535-569). Naucalpan de Juárez (México): Pearson.

Mateus, J.C., Aran-Ramspott, S, y Masanet, M-J. (2017). Revisión de la literatura sobre dispositivos móviles en la universidad española. RIED. Revista Iberoamericana de Educación a Distancia, 20(2), 49-72. http://dx.doi. org/10.5944/ried.20.2.17710

Ophir, E.; Nass, C., y Wagner, A. D. (2009). Cognitive control in media multitaskers. PNAS, 106(37), 15583-15587. https://doi.org/10.1073/pnas.0903620106

Orozco, G. (1994). Al rescate de los medios. México: Fundación Manuel Buendía Universidad Iberoamericana.

Osuna-Acedo, S., Marta-Lazo, C. Y Frau-Meigs, D. (2018). From sM00C to tM00C, learning towards professional transference. ECO European Project. Comunicar, 25(55), 105-114. https://doi.org/10.3916/C55-2018-10

Pereira, S., Fillol, J. y Moura, P. (2019). El aprendizaje de los jóvenes con medios digitales fuera de la escuela: De lo informal a lo formal. Comunicar, 27(58), 41-50. https://doi.org/10.3916/C58-2019-04

Pérez-Escoda, A., García-Ruiz, R. y Aguaded-Gómez, I. (2018). La competencia mediática del profesorado universitario. Validación de un instrumento de evaluación. @tic Revista d'innovació educativa, (21), 1-9. Recuperada de https://bit.ly/2JEHQ5n

Pérez-Rodríguez, A., Aguaded, J.I. y Monescillo, M. (2010). Hacia una integración curricular de las TIC en los centros educativos andaluces de Primaria y Secundaria. Bordón, 62(4), 7-23. 
Las dimensiones de la competencia mediática en estudiantes universitarios españoles $\mid 243$

MONOGRÁFICO

Pérez-Rodríguez, A. y Delgado-Ponce, A. (2012). De la competencia digital y audiovisual a la competencia mediática: dimensiones e indicadores. Comunicar, 20(39), 25-34. https://doi.org/10.3916/C39-2012-02-02

Pérez Tornero, J.M. (1994). El desafio educativo de la televisión: para comprender y usar el medio. Barcelona: Paidós.

Pérez-Tornero, J.M. (2004). Promoting Digital Literacy. Understanding Digital Literacy. Unión Europea: Educación y Cultura.

Perrenoud, P. (2004). Diez nuevas competencias para enseñar. Barcelona: Graó.

Quiroz, M.T. (2008). La edad de la pantalla. Tecnologías interactivas y jóvenes peruanos. Lima: Fondo Editorial Universidad de Lima.

Ramírez, F. (2015, 6 de abril). Entrevistas Grupales: Los Focus Group Vs Los Grupos de Discusión. Manual del Investigador [Blog Internet]. Recuperado de https:// bit.ly/2vXiqrC

Ramírez García, A., Sánchez-Carrero, J. y Contreras-Pulido, P. (2016). Media competence in primary education in the Spanish context. Educação e Pesquisa, 42(2), 375-394. http://dx.doi.org/10.1590/S1517-9702201606143127

Spitzer, M. (2013). Demencia digital. El peligro de las nuevas tecnologías. Barcelona: Ediciones B.

Toffler, A. (1980). La tercera ola (A. Martín, trad.). Barcelona: Plaza \& Janés. Tondeur, J., Van Braak, J. \& Valcke, M. (2007). Curricula and the use of ICT in education: Two worlds apart? British Journal of Educational Technology, 38(6), 962-976. https://doi.org/10.1111/j.1467-8535.2006.00680.x

Tucho, F., Masanet, M.J. y Blanco, S. (2014). La cuestión medioambiental en la educación mediática: un reto pendiente. Zer, 36, 205-219. Recuperado de https://bit.ly/30qa0vZ

Tucho, F., Vicente-Mariño, M. y García de Madariaga, J.M. (2017). La cara oculta de la sociedad de la información: el impacto medioambiental de la producción, el consumo y los residuos tecnológicos. Chasqui, (136), 45-61. http://dx.doi. org/10.16921/chasqui.v0i136.3321

Tyrer, C. (2019). Beyond social chit chat? Analysing the social practice of a mobile messaging service on a higher education teacher development course. International Journal of Educational Technology in Higher Education, 16(1), 13. https://doi.org/10.1186/s41239-019-0143-4 
Uncapher, M. R.; Lin, L.; Rosen, L. D.; Kirkorian, H. L.; Baron, N. S.; Bailey, K., ... Wagner, A.D. (2017). Media Multitasking and Cognitive, Psychological, Neural, and Learning Differences. Pediatrics, (140) (Supplement 2). Recuperado de https://bit.ly/2JJ0S98

Uncapher, M. R. y Wagner, A. D. (2018). Minds and brains of media multitaskers: Current findings and future directions. PNAS, 115(40), 9889-9896. https://doi. org/10.1073/pnas.1611612115

\section{Notas}

[1] En los resultados se especifica la pregunta correspondiente a cada dimensión de la competencia mediática, profundizando más en su significado.

\section{(c) (i)}

Este obra está bajo una licencia de Creative Commons Reconocimiento 4.0 Internacional. 